\title{
Risk of thromboembolism in patients developing critical illness-associated atrial fibrillation
}

\author{
Authors: Benjamin Clayton, ${ }^{A}$ Susan Ball, ${ }^{B}$ James Read ${ }^{C}$ and Sam Waddy
}

\begin{abstract}
Although common, the long-term significance of developing atrial fibrillation (AF) during a period of critical illness is unclear. We undertook a retrospective cohort analysis to assess the rate of thromboembolism (TE) in patients developing atrial fibrillation de novo during admission to our intensive care unit. In total, 1,955 patients were followed up (maximum follow-up 1,276 days) for the occurrence of TE, of which $220(11.3 \%)$ had developed AF or atrial flutter during their critical care admission. There were $11 \mathrm{TE}$ events among the patients with new AF ( 0.053 events per patient-year), compared with 18 in the non-AF group ( 0.0059 events per patient-year). The unadjusted hazard ratio for TE in patients developing new AF compared with those not developing AF was 8.09 (95\% CI 3.08-17.19, $\mathrm{p}<0.001)$. In patients admitted to critical care, the development of AF appears to be associated with a significantly increased risk of subsequent thromboembolism.
\end{abstract}

KEYWORDS: Atrial fibrillation, critical care, intensive care, critical illness, stroke, thromboembolism

\section{Introduction}

Atrial fibrillation (AF) is prevalent in acute illness, representing the commonest arrhythmia in critical care settings, ${ }^{1}$ occurring in $10-15 \%$ of patients. The physiological insult of acute illness, altered sympathetic tone or inflammation have all been proposed as triggers. ${ }^{2,3}$ The development of $\mathrm{AF}$ in this situation is a poor prognostic sign, ${ }^{4}$ particularly in severe sepsis, where it is associated with a two-fold increase in the risk of death over comparable patients who remain in sinus rhythm. ${ }^{3}$ Its prevalence, treatment and implications for mortality have therefore been extensively studied in this setting.

In the general population AF confers significant risk of ischemic stroke, ${ }^{5}$ with clear international consensus about

Authors: A specialist registrar in cardiology, Derriford Hospital, Plymouth, UK; ${ }^{B}$ NIHR CLAHRC South West Peninsula (PenCLAHRC), University of Exeter Medical School, University of Exeter, Exeter, UK; ${ }^{C}$ speciality registrar in geriatric and general internal medicine, Derriford Hospital, Plymouth, UK; ${ }^{\mathrm{D}}$ Clinical director, Intensive Care, Derriford Hospital, Plymouth, UK anticoagulant prophylaxis in all but the lowest-risk patients. ${ }^{6}$ The thromboembolic (TE) implications of AF arising during acute illness are less well-studied ${ }^{7}$ and generally time-limited to the acute phase or hospital stay. ${ }^{8,9}$ Patients in the general population experiencing paroxysmal AF carry equivalent risk to those with persistent or permanent $\mathrm{AF}^{10}$ and paroxysmal $\mathrm{AF}$ is common in patients with apparently idiopathic stroke. ${ }^{11,12}$ Furthermore, it is unclear if the TE risk of AF occurs due to the arrhythmia itself, or associated inflammatory or metabolic processes, such that the ephemeral nature of an arrhythmia may be falsely reassuring.

Critical illness brings a plethora of intrinsic and iatrogenic stimuli for arrhythmogenesis, which are more transient than traditional risk factors, but limited evidence suggests important short- and longer-term risk nonetheless. ${ }^{9}$ Therefore, this study sought to assess the risk of thromboembolism following critical illnessassociated AF arising in our Intensive Care Unit (ICU).

\section{Methods}

The study was undertaken at a single, large, UK teaching hospital with tertiary specialties. The critical care unit comprises 13 general intensive care beds, nine for neurosurgery/trauma and additional high-dependency capacity. A separate cardiothoracic ICU provides perioperative care for cardiac surgery and was not included in this study.

An electronic patient record system (Innovian, Dräger Medical, Germany) maintains real-time recording of vital statistics and note-keeping. Heart rhythm is manually inputted hourly and acute rhythm changes are flagged by nursing staff, with 12-lead electrocardiograms recorded electronically for physician review. Patients have electronic pro forma completed at admission and discharge. An independently populated electronic discharge record is used for all hospital admissions. A picture archiving and communication system (PACS) contains all imaging studies and reports. Letters from outpatient clinic visits are recorded on central computer databases. The patient administration system (PAS) records all hospital episodes including admissions, clinic attendances and deaths.

The electronic records of all patients admitted to our ICU from September 2009 to September 2011 were screened for the onset of AF or atrial flutter of at least 30 seconds duration during their admission. Exclusion criteria included patients with previously documented atrial arrhythmia, those known to have had heart valve surgery or with significant mitral valve disease, ${ }^{13,14}$ patients admitted to or discharged from ICU on therapeutic 
anticoagulation, and those less than 18 years old. Patients resident outside the hospital catchment area, where follow-up could not be reliably conducted, were also excluded. Where patients were admitted to ICU more than once in the study period, the episode in which AF was first documented was used as the index admission.

Baseline characteristics and episode details (including length of ICU stay, death and place of death if relevant, and illness severity described by Acute Physiology and Chronic Health Evaluation II [APACHE II] score ${ }^{15}$ ) were recorded. The occurrence of TE (stroke, transient ischaemic attack or systemic embolism) and presence of risk factors (heart failure, hypertension, age, diabetes mellitus, previous systemic TE, vascular disease, gender) were identified based on previously described definitions ${ }^{14}$ and a $\mathrm{CHADS}_{2}$ VASC score was calculated. TE complications were only included in the analysis if they occurred after both the onset of AF and admission to critical care, and were diagnosed by a consultant physician, or confirmed with appropriate imaging, or operative or post-mortem examination. Where there was any doubt as to the occurrence of an event, this was included as an event in the non-AF group, but excluded in the AF group.

Follow-up was conducted from the date of onset of AF, or the date of admission to the intensive care unit in patients who did not develop AF, to the earliest of: date of death, date of commencing therapeutic oral anticoagulation, or termination of study followup.

\section{Objectives and analyses}

The objective was to describe the rate of TE complications in patients developing new onset AF (or atrial flutter) in the context of acute illness or injury, in a general ICU, compared to those without AF.

We used descriptive statistics to describe the baseline characteristics of the patient population. Categorical variables were expressed as numbers (percentages). Continuous variables were expressed as means (standard deviation) or medians (25th, 75th percentiles). Event rates were described as both absolute values and per patient-year in both AF and non-AF groups. We used Kaplan-Meier estimates to show probability of TE in the two groups and Cox proportional hazards models to analyse time to TE. We used Kaplan-Meier estimates and a log-rank test to compare mortality between the two groups and Cox proportional hazards (PH) models to analyse time to death. For the main outcome, time to TE, as well as for mortality, unadjusted analysis was based on a Cox $\mathrm{PH}$ model including group (AF or non-AF) as a single predictor variable. The participant baseline characteristics age, gender, $\mathrm{CHADS}_{2}$ VASc score and APACHE II score were included as covariates in separate Cox models and any found to be individually significantly associated with the outcome were included as confounders in an adjusted Cox PH model. Results are reported as estimated HRs, with $95 \%$ confidence intervals and $\mathrm{p}$-values. All analyses were conducted using statistical software $\mathrm{R}$ (version 3.3.2). ${ }^{16}$

\section{Ethical review}

The project was reviewed by our institutional review board and registered locally as a clinical audit. Further ethical review and the need for informed consent were waived. Identifiable patient data were utilised only to facilitate the cross referencing of data sources

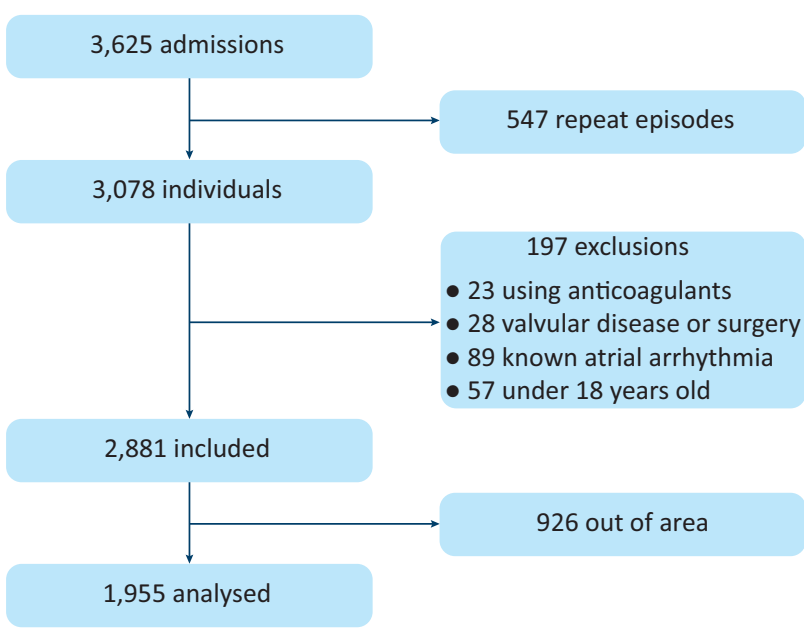

Fig 1. Patient inclusion and exclusion.

and records were otherwise anonymous. The study was conducted retrospectively, patients were not involved in its conduct and there was no impact on their care.

\section{Results}

From September 2009 to September 2011, there were 3,625 admissions to critical care, comprising 3,078 individuals. After exclusions, 1,955 patients were included in the analysis (Fig 1). Of these, $220(11.3 \%)$ developed AF or atrial flutter during their critical care admission. The baseline characteristics of both groups are described in Table 1.

\section{Thromboembolic events}

There were 11 TEs in patients with new onset AF $(0.053$ events per patient-year; mean follow-up 344 days) and 18 events in those without ( 0.0059 events per patient-year; mean follow-up 638 days). In the AF group, one patient suffered ischaemic bowel and the remainder had thrombotic strokes or transient ischaemic attacks. Two patients suffered watershed cerebral infarcts following subarachnoid haemorrhage and one during a carotid endarterectomy, all in the group without AF.

At 1 year, Kaplan-Meier estimates of the probability of TE were 0.081 (95\% CI 0.03-0.13) in the AF group, compared with 0.008 (95\% CI 0.003-0.013) in the no AF group. At 3 years, these estimates were 0.097 (95\% CI 0.037-0.152) and 0.015 (95\% CI 0.008-0.023), respectively (Fig 2).

Cox regression analysis showed strong evidence of an effect of new onset AF on time to occurrence of TE. Fitting a model with a single predictor variable for new onset $A F$, the estimated $H R$ for TE in patients with new onset AF compared to those without was 8.09 (95\% CI 3.08-17.19, p<0.001). The baseline characteristics $\mathrm{CHADS}_{2}$ VASc score, APACHE II score and age were found to be individually associated with occurrence of TE and so an adjusted analysis consisted of a Cox regression model including a variable for new onset AF and for $\mathrm{CHADS}_{2}$ VASc score, APACHE II score and age as possible confounding variables. This gave an estimated adjusted HR for TE (new onset AF / no new AF) 5.91 (95\% CI 2.60-13.44, $p<0.001)$. 
Table 1. Baseline characteristics of the study population

\section{$\mathrm{AF}(\mathrm{N}=220)$}

Male, $n(\%)$

Age, in years, median (25th, 75th percentile)

Baseline risk factors, $\mathrm{n}(\%)$

$>$ Heart failure

$>$ Hypertension

$>$ Diabetes mellitus

> Stroke or systemic embolism

> Vascular disease

$\mathrm{CHADS}_{2}$ VASc score, mean (SD)

APACHE score, mean (SD)
137 (62.3)

$73.0(64.0,80.0)$

$20(9.1)$

$110(50.0)$

35 (15.9)

$24(10.9)$

$64(29.1)$

$2.8(1.6)$

$20.7(7.0)$
No AF ( $\mathrm{N}=1735)$

901 (51.9)

$62.0(47.0,72.0)$

$59(3.4)$

$79(4.0)$

390 (22.5)

500 (25.6)

237 (12.1)

$110(5.6)$

327 (16.7)

1.8 (1.5)

$15.1(7.4)$

$\mathrm{AF}=$ atrial fibrillation; $\mathrm{APACHE}=$ Acute Physiology and Chronic Health Evaluation II score; SD = standard deviation

\section{Mortality}

The mortality rate for the total follow-up period was $61.8 \%$ (136/220) in the group with new AF compared to $33.9 \%$ $(589 / 1,735)$ in the group without. Analysis of time to death showed evidence of increased mortality among patients developing new $A F$, compared to those who did not $(p<0.001$ log-rank test). At 1 year, Kaplan-Meier estimates of survival were 0.45 (95\% CI 0.38-0.52) in the AF group, compared with 0.72 (95\% CI $0.70-0.74)$ in the no AF group. At 3 years, these estimates were 0.35 (95\% CI 0.28-0.42) and 0.64 (95\% CI 0.62-0.67), respectively. In-hospital mortality rates were $47.7 \%$ (105/220) and $19.3 \%(335 / 1,735)$, respectively. Of those with $A F, 80$ died in ICU $(36.4 \%)$ compared to $228(13.1 \%)$ of those without. Three patients died in hospital on the same admission following a TE event; all had developed new onset AF. Cox regression analysis showed strong evidence of an effect of new onset AF on mortality. Fitting a model with a single predictor variable for new onset AF, the estimated $\mathrm{HR}$ for mortality in patients with new onset AF compared to those without was 2.47 (95\% CI 2.05-2.97, $\mathrm{p}<0.001$ ). The baseline characteristics $\mathrm{CHADS}_{2}$ VASc, APACHE II, gender and age were found to be individually associated with mortality and so an adjusted analysis consisted of a Cox regression model including a variable for new onset AF and for $\mathrm{CHADS}_{2}$ VASc, APACHE II, gender and age as possible confounding variables. This gave an estimated $\mathrm{HR}$ for mortality (new onset $\mathrm{AF} /$ no new $\mathrm{AF}$ ) of 1.53 (95\% CI 1.26-1.87, p<0.001).

\section{Discussion}

This study suggests that, rather than being simply an inevitable consequence of a physiological insult, the onset of AF or flutter
Fig 2. Kaplan-Meier estimates of the probability of thromboembolism (TE) in patients with new atrial fibrillation (AF) (red line), and in those without new AF (blue line). Numbers accompanying the numbers at risk in parentheses are the cumulative number of censored observations.

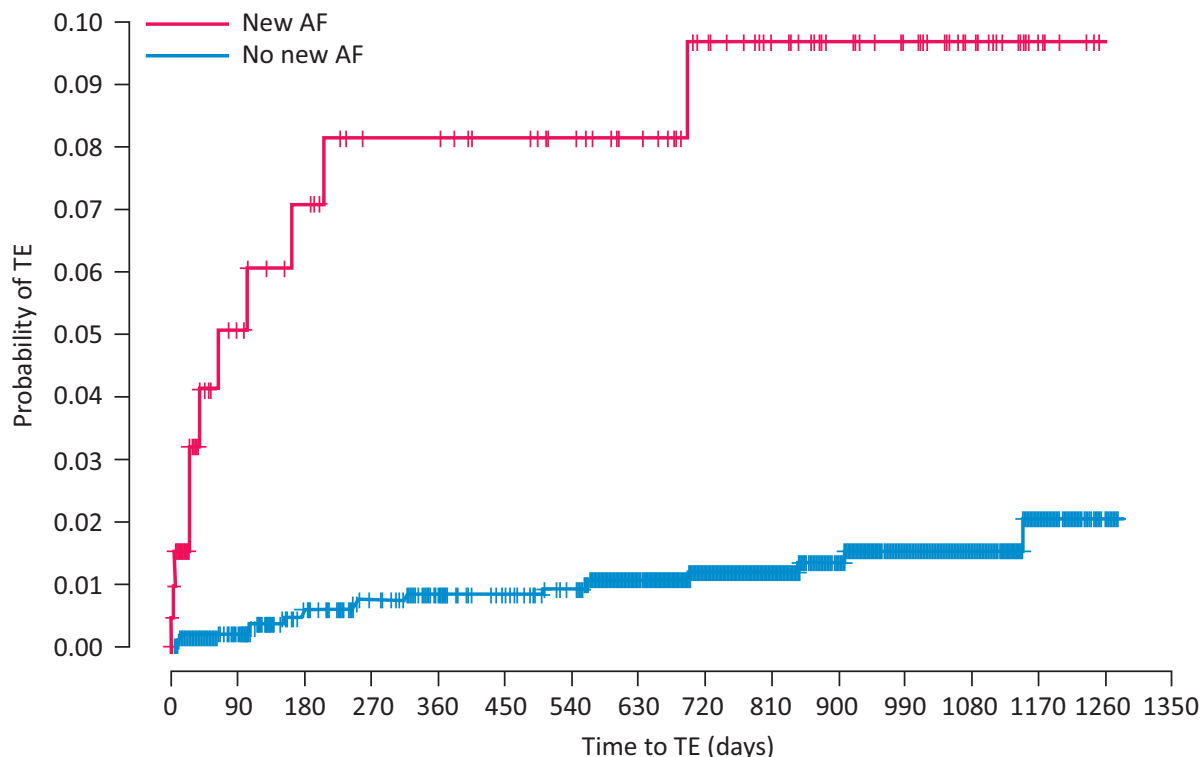

Numbers at risk

New AF $215(5) \quad 91(120) \quad 84(126) \quad 74(136) \quad 57(152) \quad 38(171) \quad 20(189) \quad 0(209)$ No new AF $1648(87) 1287(440) 1229(495) \quad 1186(537) \quad 876(844) 556(1163) 284(1434) \quad 29(1688)$ 
in the acutely ill patient is associated with an increased risk of TE complications. This risk appears to persist and may exceed that seen in the general population. ${ }^{14}$

The rate of in-hospital death in patients with AF was high in our study, a known association particularly in sepsis, ${ }^{3,4,9}$ and this finding is likely to reflect disease severity. ${ }^{17}$

The association of AF with critical illness is well established ${ }^{1}$ and appears common to all acute illness - medical patients in intensive care develop AF around day 1 or 2, similar to trauma ${ }^{18,19}$ and postoperative patients. ${ }^{20}$ The pathophysiology of AF and aetiology of thrombosis in this context are not completely understood. ${ }^{21}$ Inflammatory mediation has been proposed; acuteonset $\mathrm{AF}$ triggers platelet activation and monocyte interaction within minutes ${ }^{22}$ and platelets continue to express CD40 some weeks after successful cardioversion. ${ }^{23}$ Although the cause-effect relationship is debated, this might explain the high rates of AF and $T E$, although it is not clear whether inflammation, or some other pathology, ${ }^{24}$ persists to maintain TE risk over the duration we have observed.

\section{Limitations and other studies}

The major limitation of this study is its retrospective nature, with inherent difficulty verifying data accuracy. Although multiple sources were cross-referenced, potentially important data such as primary care records have not been examined, with the assumption that all thromboembolic complications would be referred for specialist assessment. We included patients with AF on the first day of their critical care admission if this had not been previously documented, presuming that the illness necessitating their admission also precipitated their AF, whereas it may have been present before. Pragmatically, these patients would usually be considered as having new onset AF and this therefore does not materially alter the clinical question. The small numbers of events in either group limits multivariable analysis, although the large difference between groups and concordance with other, previous studies does provide some confidence.

Anticoagulation may confound the risk assessment and followup of patients. Prophylaxis of venous thrombosis is standard practice for all critically ill patients without a clear contraindication and may have reduced the risk of in-hospital TE. Our omission of primary care data also risks failing to identify patients with AF who began anticoagulation in the community at a later date, although we would expect this to reduce the event rate in the AF group. Therefore, if such patients were excluded this may actually increase the observed difference. Further limitations include the high exclusion rate for patients out of area, which was necessary to optimise the accuracy of follow-up, and the single-centre nature of this analysis.

Most prior assessment of this issue occurred via small, short-term studies. ${ }^{7,8}$ However, recently one group has undertaken a number of large, population-based, epidemiological studies exploring the association of new-onset AF with sepsis. Walkey et al used hospital claims data to demonstrate a $2.6 \%$ risk of in-hospital stroke and $2.0 \%$ risk of readmission with stroke (non-significant trend) within the same year, highlighting the potential TE risk of new onset AF for the first time. ${ }^{9}$ A subsequent study of Medicare beneficiaries identified an increase in ischaemic stroke at 5 years from an admission with sepsis (5.3\% in those with new-onset AF vs $4.7 \%$ without; HR 1.22; 95\% CI 1.15-1.47). ${ }^{25}$ These studies acknowledge the limitations of claims data, particularly with regards to coding and the temporal association of triggers and events. This current study supports these previous analyses by providing, for the first time on this scale, patient-level analysis of all admissions through a critical care unit, incorporating history, physiological parameters, diagnoses and investigations.

One further study has considered the predictive value of $\mathrm{CHADS}_{2}$ and $\mathrm{CHADS}_{2}$ VASc scoring in the general critical care population. ${ }^{26}$ However, $25 \%$ of patients had a history of atrial arrhythmia predating their critical illness and $9 \%$ experienced atrial tachycardia, which is not universally accepted as a risk factor for thromboembolism. In the 6 months of follow-up there were 12 recorded 'thromboembolic' complications, including two myocardial infarctions and four patients with documented left atrial appendage thrombus without embolic sequelae. Finally, the use of anticoagulation beyond the critical care stay was not considered.

\section{Future questions and research}

The implication for patients outside critical care environments is less clear. Although AF is commonly precipitated by acute illness the illness severity and subsequently high mortality rates in our patients may be different to the overall hospital population, and differences in the use and availability of critical care facilities may limit the applicability of these findings to other countries. There are a plethora of aetiological factors for both AF and TE associated with critical illness, ${ }^{27}$ including inflammation, septic emboli, fluid shifts, and cardiac and vasoactive pharmacotherapy, which may be relevant for the prevention and management of both $\mathrm{AF}$ and TE, in addition to pre-existing comorbidities. ${ }^{27,28}$ We have demonstrated association rather than causality and further research is needed to establish whether the physiological insult of critical illness itself, or its management, present an independent risk of future TE. The true implication for all patients with critical illness will only be clarified with larger, prospective study.

$\mathrm{AF}$ is common following cardiothoracic surgery with a stroke risk of $2-5 \% .{ }^{29,30}$ One systematic review found no AF-reduction strategy that significantly reduced the risk of stroke. ${ }^{29}$ As with the general population, ${ }^{10}$ aggressive rhythm control does not mitigate the risk of TE, although anticoagulation post-surgery may. ${ }^{31}$ Large epidemiological studies of the management of AF in critical care imply reticence to use anticoagulants in the acute phase due to the risk of haemorrhage ${ }^{26,28}$ and lack of short-term efficacy. ${ }^{32}$ These findings reflect the general AF population, where longer term anticoagulation, and not rhythm control, is protective against $T E$. This will need to be carefully considered in future strategies for the reduction of TE in these patients.

\section{Conclusion}

This study suggests that the onset of AF, or atrial flutter, during critical illness may be associated with longer-term risk of systemic thromboembolism. Whether the occurrence of AF is causative, or a marker of increased risk, is not clear and similarly the role of mediators such as hypertension need further scrutiny, but the risk of TE appears to persist beyond the period of acute illness. Further, prospective, multicentre analysis of this association is urgently required. 


\section{What is already known about this subject?}

$>\mathrm{AF}$ is the commonest arrhythmia in critical care environments and its development during critical illness is associated with a worse prognosis.

> In the general population, the development of even paroxysmal $\mathrm{AF}$ is associated with an increased risk of systemic thromboembolism.

\section{What does this study add?}

> The development of de novo AF during a period of critical illness is associated with an increased longer term risk of systemic thromboembolism.

\section{How might this impact on clinical practice?}

> There is urgent need to establish the long-term risk to patients who have experienced AF during an acute illness.

> Strategies for risk assessment and possibly long-term anticoagulation need to be considered.

> Further consideration of the pathogenesis of thromboembolism in $\mathrm{AF}$, and the relationship between cardiac arrhythmia and systemic processes, is warranted.

\section{Author contributions}

$B C$ designed the study, undertook data collection and supplementary data analysis, wrote and revised the manuscript and approved the final version. $\mathrm{BC}$ is the guarantor of the study.

SB undertook data analysis, critically revised and contributed to the manuscript and approved the final version. JR undertook data collection and assisted with its analysis, assisted with the critical revision of the manuscript and approved the final version. SW supervised the study, criticised and amended the study design, critically revised the final article and approved the final version.

\section{Acknowledgements}

We gratefully acknowledge the contribution of Michael Eaglestone, who undertook interrogation of the electronic critical care database and assisted with data collection.

\section{Funding}

No funding of any sort has been sought by any author for the conduct of this study.

SB is funded by the National Institute for Health Research (NIHR) Collaboration for Leadership in Applied Health Research and Care (CLAHRC) for the South West Peninsula. The views expressed in this publication are those of the authors and not necessarily those of the National Health Service, NIHR or the Department of Health in England. The remaining authors have not received any funding for the conduct of this study.

\section{References}

1 Annane D, Sébille V, Duboc D et al. Incidence and prognosis of sustained arrhythmias in critically ill patients. Am J Respir Crit Care Med 2008:178:20-5.

2 Issac TT, Dokainish H, Lakkis NM. Role of inflammation in initiation and perpetuation of atrial fibrillation. A systematic review of the published data. J Am Coll Cardiol 2007;50:2021-8.
3 Meierhenrich R, Steinhilber E, Eggermann C et al. Incidence and prognostic impact of new-onset atrial fibrillation in patients with septic shock: a prospective observational study. Crit Care 2010;14:R108

4 Arora S, Lang I, Nayyar V et al. Atrial fibrillation in a tertiary care multidisciplinary intensive care unit - incidence and risk factors. Anaesth Intensive Care 2007;35:707-13.

5 Wolf PA, Abbott RD, Kannel WB. Atrial fibrillation as an independent risk factor for stroke: the Framingham Study. Stroke 1991;22:983-8.

6 Camm AJ, Kirchhof P, Lip GYH et al. Guidelines for the management of atrial fibrillation: the Task Force for the Management of Atrial Fibrillation of the European Society of Cardiology (ESC). Eur Heart ] 2010:31:2369-429.

7 Sleeswijk ME, Van Noord T Tulleken JE et al. Clinical review: treatment of new-onset atrial fibrillation in medical intensive care patients - a clinical framework. Crit Care 2007;11:233.

8 Salman S, Bajwa A, Gajic $\mathrm{O}$ et al. Paroxysmal atrial fibrillation in critically ill patients with sepsis. J Intensive Care Med 2008;23:17883

9 Walkey AJ, Wiener RS, Ghobrial JM et al. Incident stroke and mortality associated with new-onset atrial fibrillation in patients hospitalized with severe sepsis. JAMA 2011;306:2248-54.

10 Friberg L, Hammar N, Rosenqvist M. Stroke in paroxysmal atrial fibrillation: Report from the Stockholm Cohort of Atrial Fibrillation. Eur Heart ] 2010;31:967-75.

11 Flint AC, Banki NM, Ren X et al. Detection of paroxysmal atrial fibrillation by 30-day event monitoring in cryptogenic ischemic stroke: The stroke and monitoring for PAF in real time (SMART) registry. Stroke 2012;43:2788-90.

12 Rizos T, Güntner J, Jenetzky E et al. Continuous stroke unit electrocardiographic monitoring versus 24 -hour holter electrocardiography for detection of paroxysmal atrial fibrillation after stroke. Stroke 2012;43:2689-94.

13 Gage BF, Waterman AD, Shannon W et al. Validation of clinical classification schemes for predicting stroke: results from the National Registry of Atrial Fibrillation. JAMA 2001;285:286470.

14 Lip GYH, Nieuwlaat R, Pisters R et al. Refining clinical risk stratification for predicting stroke and thromboembolism in atrial fibrillation using a novel risk factor-based approach: The Euro Heart Survey on atrial fibrillation. Chest 2010:137:263-72.

15 Knaus WA, Draper EA, Wagner DP et al. APACHE II: a severity of disease classification system. Crit Care Med 1985;13:818-29.

16 R Core Team. R: A language and environment for statistical computing. 2016. www.r-project.org/

17 Carrera P, Thongprayoon C, Cheungpasitporn W et al. Epidemiology and outcome of new-onset atrial fibrillation in the medical intensive care unit. J Crit Care 2016:36:102-6.

18 Seguin P, Laviolle B, Maurice A et al. Atrial fibrillation in trauma patients requiring intensive care. Intensive Care Med 2006;32:398404.

19 Hadjizacharia P, O'Keeffe T, Brown CVR et al. Incidence, risk factors, and outcomes for atrial arrhythmias in trauma patients. Am Surg 2011:77:634-9.

20 Heinz G. Arrhythmias in the ICU: what do we know? Am J Respir Crit Care Med 2008;178:1-2.

21 Guo Y, Lip GYH, Apostolakis S. Inflammation in atrial fibrillation. J Am Coll Cardiol 2012;60:2263-70.

22 Hayashi M, Takeshita K, Inden Y et al. Platelet activation and induction of tissue factor in acute and chronic atrial fibrillation: Involvement of mononuclear cell-platelet interaction. Thromb Res 2011;128:e113-8.

23 Duygu $\mathrm{H}$, Barisik V, Kurt $\mathrm{H}$ et al. Prognostic value of plasma soluble CD40 ligand in patients with chronic non-valvular atrial fibrillation. Europace 2008;10:210-4 
24 Wijesurendra RS, Liu A, Eichhorn C et al. Lone Atrial fibrillation is associated with impaired left ventricular energetics that persists despite successful catheter ablation. Circulation 2016;134:1068-81.

25 Walkey AJ, Hammill BG, Curtis LH et al. Long-term outcomes following development of new-onset atrial fibrillation during sepsis. Chest 2014;146:1187-95.

26 Champion S, Lefort Y, Gaüzère BA et al. CHADS2 and CHA2DS2-VASc scores can predict thromboembolic events after supraventricular arrhythmia in the critically ill patients. J Crit Care 2014;29:854-8.

27 Makrygiannis SS, Margariti A, Rizikou D et al. Incidence and predictors of new-onset atrial fibrillation in noncardiac intensive care unit patients. J Crit Care 2014;29:697.e1-5.

28 Kanji S, Williamson DR, Yaghchi BM et al. Epidemiology and management of atrial fibrillation in medical and noncardiac surgical adult intensive care unit patients. J Crit Care 2012;27:326.e1-8.

29 Arsenault KA, Yusuf AM, Crystal E et al. Interventions for preventing post-operative atrial fibrillation in patients undergoing heart surgery. Cochrane database Syst Rev 2013;1:CD003611.
30 Almassi GH, Schowalter T, Nicolosi AC et al. Atrial fibrillation after cardiac surgery: a major morbid event? Ann Surg 1997;226: 501-11.

31 Hata M, Akiyama K, Wakui S et al. Does warfarin help prevent ischemic stroke in patients presenting with post coronary bypass paroxysmal atrial fibrillation? Ann Thorac Cardiovasc Surg 2013;19:207-11.

32 Walkey AJ, Quinn EK, Winter MR et al. Practice patterns and outcomes associated with use of anticoagulation among patients with atrial fibrillation during sepsis. JAMA Cardiol 2016;1:682-90.

Address for correspondence: Dr Benjamin Clayton, Cardiology Department, Derriford Hospital, Plymouth PL6 8DH, UK.

Email: benjamin.clayton@nhs.net

\section{NGC

\section{New to systematic reviewing, or need to improve your critical appraisal skills?}

Our intensive 1-day courses will introduce you to the key principles of critical appraisal and systematic reviewing, and equip you with the basic skills to put your knowledge into practice.

> Introduction to critical appraisal

> Systematic reviews and meta-analysis in action

Discount available if both courses are booked together.

\section{'Informative and practical'} Dr Rajeswari Ramaraj

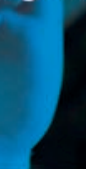

CPD credits

For course dates and more information visit: www.ngc.ac.uk/training-courses 